\title{
Selección léxica y encuadre conceptual en el discurso de los progresistas españoles respecto a los debates de género de la XII legislatura (2016-2019)
}

\section{Lexical selection and conceptual framing in the discourse of the Spanish progressives regarding the gender debates of the XII legislature (2016- 2019)}

\author{
Carmen González Gómez \\ Universidad de Salamanca, Salamanca \\ carmen.gonzalez@usal.es
}

ACCESO ABIERTO / OPEN ACCESS

Cita: González Gómez, Carmen (2021). Selección léxica y encuadre conceptual en el discurso de los progresistas respecto a los debates de género de la XII legislatura (2016-2019). Textos en Proceso, 7(2), pp. 83-95.

https://doi.org/10.17710/tep.2021.7. 2.6gonzalezgomez

Editoras: Esperanza Alcaide Lara (Universidad de Sevilla) y Ana Pano Alamán (Università di Bologna)

Recibido: 30/09/2021

Aceptado: 10/12/2021

Conflicto de intereses: La autora ha declarado que no posee conflicto de intereses.

Copyright: (c) Carmen González Gómez. Esta obra está bajo licencia Creative Commons Reconocimiento 4.0.

\section{Resumen}

Este estudio analiza el discurso parlamentario sobre igualdad de género elaborado por el PSOE y Unidos Podemos durante la XII legislatura (2016-2019) del parlamento español. La investigación parte de un corpus constituido por 77.828 palabras extraídas de los treinta debates plenarios que abordaron cuestiones relacionadas con la igualdad de género durante la XII legislatura. El estudio combina la perspectiva cuantitativa y cualitativa para analizar si se dan asimetrías en el uso de términos como feminismo, igualdad, cuidados o machismo. El análisis de 30 voces pone de manifiesto que existen diferencias entre ambos partidos y que es Unidos Podemos quien emplea con más frecuencia la mayor parte de los términos. Esta aproximación cuantitativa se completa con un análisis cualitativo que aborda qué marcos activan las voces en cuestión. El objetivo de esta investigación es dar cuenta de cómo utilizan las formaciones progresistas los principales términos en boga en los debates sobre igualdad de género.

Palabras clave: análisis del discurso, discurso político, selección léxica, marco conceptual, igualdad, género.

\section{Abstract}

This study analyzes the parliamentary discourse on gender equality made by PSOE and Unidos Podemos during the XII legislature (2016-2019). The research is based on a corpus made up of 77.828 words extracted from the thirty plenary debates that 
addressed issues related to gender equality during the XII legislature. The study combines the quantitative and qualitative perspectives to analyze whether there are asymmetries in the use of terms such as feminism, equality, cuidados or machismo. The analysis of the use of 31 lexical items shows that there are differences between the two parties, and that Unidos Podemos is the one that most frequently uses this kind of terms. This quantitative approach is completed with a qualitative study that analyzes which frames activate these words. The objective of this research is to give an account of how progressive political parties use the main terms in vogue in debates about gender equality. The main goal is, therefore, to see how ideology influences the lexical selection of political discourse.

Keywords: discourse analysis, political discourse, lexical selection, conceptual framework, equality, gender.

\title{
1. Introducción: el contexto sociopolítico
}

\author{
"Si la mujer tiene derecho a subir al cadalso, \\ también debe tener derecho a subir a la tribuna" \\ Olympe de Gouges, Declaración de los derechos de la mujer y la ciudadana, \\ artículo X, 1791
}

Las cuestiones relacionadas con la igualdad de género interesan a la política española desde hace al menos dos décadas. Existe hoy en día un consenso institucional amplio en torno a la lucha contra la discriminación y la violencia machista. Esta conformidad ha dado lugar a grandes acuerdos parlamentarios que han contado con el apoyo unánime de la cámara. Los más destacados han sido la Ley Orgánica de Medidas de Protección Integral contra la Violencia de Género, nacida durante el gobierno progresista de Zapatero y aprobada en el Congreso por unanimidad a finales de 2004 -fue entonces la primera ley integral contra la violencia de género de Europa-; y, en segundo lugar, el Pacto de Estado contra la violencia machista, promulgado durante el gobierno conservador de Rajoy a finales de 2017. Fue ratificado en la cámara baja por todas las formaciones políticas, a excepción de Unidos Podemos ${ }^{1}$, que se abstuvo al considerar que las medidas pactadas eran insuficientes.

De forma paralela a las reformas legislativas se han producido transformaciones sociales. Desde 2010, tienen lugar, en diversos países del mundo occidental, multitudinarias manifestaciones en favor de la igualdad de género. Este incremento en la movilización ciudadana ha llevado a considerar el nacimiento de una cuarta ola feminista ${ }^{2}$. Forman parte de esta corriente colectivos como "Ni Una

\footnotetext{
${ }^{1}$ En la actualidad el partido se denomina Unidas Podemos, pero nos referimos a él por su nombre durante la XII legislatura (2016-2019).

${ }^{2}$ De manera tradicional, se distinguen tres períodos históricos - denominados a menudo olas- en los que el feminismo adquiere especial relevancia. El primero de ellos se extiende desde la Revolución Francesa hasta mediados del siglo XIX y reivindica la ciudadanía de pleno derecho de las mujeres y el acceso a la educación. El segundo comienza en 1848, con la convención de Seneca Falls, la primera
} 
Menos", nacido en Argentina en 2015 contra los feminicidios, o movimientos como "Me too", surgido en 2017 en Hollywood a raíz de las acusaciones de acoso sexual contra el productor Harvey Weinstein. En nuestro país, la eclosión de este auge es visible a partir de 2018.

Estas transformaciones han tenido en buena medida un carácter discursivo. Han implicado neología, cambios semánticos y resignificación de la realidad. La nueva forma de nombrar ha permeado igualmente el discurso político; en la XII legislatura están presentes voces como cuidados, patriarcado, corresponsabilidad o sexoafectivo. Por tanto, la esfera discursiva no solo adquiere relevancia en los momentos históricos (De Santiago, 1992, pp. 16-17; Fernández Lagunilla, 1999b, p. 57), sino que participa y contribuye a través del caudal léxico al cambio sociopolítico.

\section{Selección léxica y frames: el poder mágico de la palabra}

La selección léxica es una herramienta fundamental en la estrategia política (De Santiago, 2005, p. 109). Si los términos elegidos importan no es tanto porque el lenguaje político se caracterice por la utilización de cierto léxico específico (Gallardo, 2004, p. 49), sino más bien porque las voces implicadas en el debate revelan imaginarios políticos. Desde los supuestos de la lingüística cognitiva, la reiteración de los términos instaura un encuadre conceptual que condiciona la percepción de la realidad. La jerga política no constituye una terminología cerrada (Fernández Lagunilla, 1999, p. 11), sino un corpus léxico que en ciertos casos se ideologiza. A diferencia del acervo común, donde la relación entre el significado y el significante tiende a ser estable, el léxico político es volátil y cambia de significado con relativa rapidez. De Santiago y López Eire (2000, p. 109) denominan poder mágico a esta característica de la palabra política.

La interfaz léxico-ideológica del lenguaje político ha sido estudiada por el Análisis crítico del discurso (ACD) y por la lingüística cognitiva. Desde el primer enfoque se han abordado las relaciones de poder y discriminación encubiertas en las estructuras lingüísticas (Van Dijk, 2001, p. 352; Wodak, 2003, p. 19); desde el segundo, se ha descrito la conceptualización como un proceso de encuadre a partir de marcos semánticos. Los temas preeminentes de este binomio lenguaje-ideología han sido la construcción de la identidad, el nacionalismo o las prácticas discriminatorias. En esta última parcela destacan, en el caso español, los estudios sobre lenguaje político y derechos LGTBI de Arrieta (2017 y 2021), los de Martín Rojo y Van Dijk (2007), Zapata-Barrero (2007) y Olmos (2012) sobre racismo o el de Francesconi (2012) sobre la representación del género en el discurso.

Tanto el ACD como la lingüística cognitiva han mostrado interés por la relación que mantienen la identidad de género y la palabra. Por citar solo algunos

en abordar los derechos femeninos. Aquí se sitúa también el nacimiento del sufragismo, que tuvo especial relevancia en la Inglaterra de principios de siglo XX, en torno a figuras como Pankhurst o Davison. La tercera ola se inicia en los años sesenta del siglo pasado, vinculada al mayo del 68 francés y a los procesos políticos y culturales que tienen lugar en Estados Unidos. Se cuestiona en este momento la existencia de una sociedad patriarcal que da lugar a asimetrías de género estructurales y que incide en los estereotipos sexistas. Una de las obras de referencia en este momento es El segundo sexo de Simone de Beauvoir. 
ejemplos, Tannen (1994) y Litosseliti y Sunderland (eds.) (2002) exploran cómo se reflejan las asimetrías de género en el lenguaje; en la tradición cognitiva, Lakoff y Johnson (1987) analizan los marcos sexistas que llevan a considerar la violación como un proceso que se desencadena de forma natural e irreversible.

Esta investigación cuestio la relación entre género y discurso político, pero no se encuadra específicamente en la tradición de los ACD. No tiene como cometido reflexionar sobre el sexismo discursivo ni sobre la discriminación latente en el lenguaje político. Por otra parte, entiende el concepto de framing desde la lingüistica comunicativa y no desde la teoría de la comunicación (Ardèvol-Abreu, 2015), enfoque que no descartamos en futuros estudios. Esta investigación es más bien continuadora de los estudios lexicológicos que, en la línea de García Santos (1980, 1982), Fernández Lagunilla (1999a, 1999b), De Santiago (1992), Núñez Cabezas (2000), Núñez Cabezas y Guerrero Salazar (2002) o Del Rey Morató (2011), se interesan por los corpus léxicos que caracterizan el habla de los políticos.

El nivel semántico es, junto el pragmático, el más estudiado porque la selección léxica es una herramienta central de la estrategia electoral. Como ha mostrado la cognición política, la reiteración de ítems o de ciertas ideas en forma de eslogan es eficaz por cuanto permite al electorado sentirse identificado con un partido. La elección de los términos no es, por tanto, azarosa, sino que persigue la activación de un marco mental que influya en la opinión pública. Panncake (1993) sostiene, por ejemplo, que las metáforas difundidas por la administración Bush contribuyeron a que la sociedad se mostrase favorable a la intervención militar del Golfo Pérsico. En la misma línea, Lakoff (2004, p. 69) ha señalado que los republicanos enmarcaron hábilmente la invasión iraquí para hacerla parecer una guerra justa que se libraba contra un tirano en aras de restaurar la democracia.

En el discurso político sobre igualdad de género, la selección léxica condiciona asimismo la percepción del debate. No se activa el mismo marco cuando se alude a la desigualdad que cuando se habla de patriarcado; hay diferencia entre referirse a la violencia doméstica y hacerlo a la violencia machista; las voces conciliación y cuidados apuntan a un esquema mental distinto; y aunque igualdad y feminismo tengan el mismo significado denotativo, las connotaciones que despiertan son diferentes. Esta investigación esclarece qué voces relativas al debate de género emplean las dos formaciones progresistas en el discurso parlamentario de la XII legislatura y cuáles son los principales marcos en boga.

\section{Objetivos y metodología}

Este estudio parte de un corpus constituido por los treinta debates plenarios que abordaron en la XII legislatura (2016-2019) temas relacionados con la igualdad de género. La muestra total se compone de 77.828 palabras desglosadas del siguiente modo: PSOE, 35.703 palabras y Unidos Podemos, $42.125^{3}$. El objetivo principal es conocer cómo emplean las dos formaciones progresistas 30 términos relacionados con la igualdad de género.

\footnotetext{
${ }^{3}$ La ligera diferencia entre el número de palabras de los dos corpus se debe a que los diputados de Unidos Podemos han realizado intervenciones parlamentarias más extensas.
} 
La investigación constituye un trabajo preliminar sobre la selección léxica en los debates de género en el discurso parlamentario de los progresistas. Para ello, lleva a cabo un estudio cuantitativo de las frecuencias absolutas con el fin de conocer qué voces explotan de manera más habitual los dos partidos. Los objetivos específicos son comprobar si existen asimetrías condicionadas por la ideología, si hay voces de consenso que utilicen ambas formaciones o si, por el contrario, existen palabras que alguno de los partidos evita. Tras esta aproximación cuantitativa se procede al análisis cualitativo. Se estudia el encuadre conceptual activado con los términos y se indaga en los marcos que se consolidan a través de la selección léxica. Con ello se ofrece una aproximación que espera servir de base a futuros trabajos sobre el léxico político a propósito de la igualdad de género.

\section{Análisis}

Este estudio considera 30 términos relacionados con la igualdad de género puestos en boga durante la XII legislatura ${ }^{4}$. Incluye las voces acoso, agresión, asesinato, brecha laboral, brecha salarial, conciliar, corresponsabilidad, cuidados, desigualdad, discriminación, estructural, feminismo, hermanas, igualdad, machismo, maltrato, maternidad, miedo, mujeres, nosotras, patriarcado, permisos, pobreza, precariedad, revolución, terrorismo, víctima, violación, violencia, violencias. Para cada entrada tiene en cuenta tanto los propios términos como sus derivados (no solo asesinato sino también asesinadas o asesinar) ${ }^{5}$.

\subsection{Análisis cuantitativo}

La comparación de frecuencias absolutas constata que la formación morada utiliza en más ocasiones que el PSOE voces como feminismo, patriarcado, violencias, cuidados, miedo, violación, estructural, revolución, nosotras o terrorismo. El término feminismo, pese a estar cada vez más afianzado con la acepción primera que recoge el $D L E$ ("principio de igualdad de derechos de la mujer y el hombre"), no abunda en el corpus de los socialistas, que prefieren la voz igualdad. Esto se debe a que el término continúa estando más marcado desde el punto de vista ideológico, a pesar de que en determinados contextos puede tener un sentido equivalente al de igualdad. Véase la comparación de frecuencias absolutas:

Tabla 1. XXX

\begin{tabular}{l|c|c} 
& U. Podemos & PSOE \\
\hline feminismo & 111 & 9 \\
\hline igualdad & 100 & 78 \\
\hline
\end{tabular}

El término patriarcado es utilizado de forma predominante por Unidos Podemos; de hecho, está ausente en las intervenciones del PSOE. Violencias, en plural, es otra de las voces difundidas por la formación morada, para aludir a todas

\footnotetext{
${ }^{4}$ Esta lista se configura después de constatar en una primera criba que son los términos relacionados con el debate de género más utilizados durante la legislatura.

${ }^{5}$ A la hora de realizar la selección se consideran solo los términos que se utilizan en sentido recto y no dentro de otras expresiones. Así, la voz género no se tiene en cuenta cuando aparece en "Ley contra la Violencia de Género", e igualdad no se contabiliza en la construcción "Ministra de Igualdad".
} 
las formas de discriminación sexista; no se atestigua tampoco en el discurso del PSOE. En cuanto a machismo, aparece en los discursos de ambas fuerzas, aunque sobresale en el corpus de Unidos Podemos.

Tabla 2. XXX

\begin{tabular}{|l|c|c|} 
& U. Podemos & PSOE \\
\hline patriarcado & 11 & 0 \\
\hline machismo & 109 & 51 \\
\hline violencias & 21 & 0 \\
\hline
\end{tabular}

Otros tres términos empleados de manera recurrente por el partido que lideraba Pablo Iglesias son revolución, que participa del esquema bélico y entiende el feminismo como un proceso transformador; terrorismo, empleado para conceptualizar los asesinatos machistas como atentados terroristas que se derivan de la estructura patriarcal; y estructural, utilizado para defender que el machismo es un problema sistémico que afecta a todos los órdenes.

Tabla 3. XXX

\begin{tabular}{|l|c|c|} 
& U. Podemos & PSOE \\
\hline revolución & 11 & 1 \\
\hline terrorismo & 4 & 0 \\
\hline estructural & 13 & 5 \\
\hline
\end{tabular}

Las voces acoso y agresión son asimismo empleadas de forma mayoritaria por Unidos Podemos. Ambas tienen connotaciones negativas y subrayan la violencia a la que están expuestas las mujeres. Lo mismo sucede con palabras como asesinato, maltrato, miedo, víctima, violación y violencia. Se atestiguan en el corpus parlamentario del PSOE, pero en menor medida que en el de Unidos Podemos.

Tabla 4. XXX

\begin{tabular}{|l|c|c|}
\hline & U. Podemos & PSOE \\
\hline Acoso & 5 & 1 \\
\hline agresión & 20 & 6 \\
\hline asesinato & 30 & 21 \\
\hline maltrato & 5 & 3 \\
\hline Miedo & 15 & 3 \\
\hline violación & 17 & 1 \\
\hline víctima & 43 & 32 \\
\hline violencia & 119 & 101 \\
\hline
\end{tabular}

En el campo semántico relacionado con la conciliación, los términos corresponsabilidad, permisos y conciliación se utilizan con una frecuencia similar en ambos partidos. Los dos primeros destacan en el discurso de los socialistas. La voz cuidados es característica del discurso parlamentario de Unidos Podemos. Con ella se critica que las mujeres asuman, generalmente, la mayor parte del trabajo doméstico, o el cuidado a cargo de personas dependientes, de forma no remunerada. El partido también destaca en el uso de la voz maternidad.

Tabla 5. XXX 


\begin{tabular}{|l|c|c|}
\hline conciliar & 19 & 19 \\
\hline corresponsabilidad & 12 & 15 \\
\hline cuidados & 49 & 7 \\
\hline maternidad & 25 & 8 \\
\hline permisos & 29 & 35 \\
\hline
\end{tabular}

De igual modo se observan asimetrías en el empleo de términos como hermanas, utilizado de manera exclusiva por Unidos Podemos; y de mujeres y nosotras, sustantivos con los que se apela a la población femenina y que utiliza de forma predominante el partido morado.

Tabla 6. XXX

\begin{tabular}{|l|c|c|} 
& U. Podemos & PSOE \\
\hline hermanas & 8 & 0 \\
\hline mujeres & 409 & 162 \\
\hline nosotras & 75 & 12 \\
\hline
\end{tabular}

En el léxico relacionado con la desigualdad salarial o con la discriminación también aparecen diferencias reseñables: ambas formaciones prefieren la construcción brecha salarial a brecha laboral, aunque la expresión destaca de forma clara en el discurso de Unidos Podemos. Lo mismo sucede con los términos pobreza y precariedad, más explotados por este y empleados para plantear que la desigualdad de género repercute en el terreno económico.

Tabla 7. XXX

\begin{tabular}{|l|c|c|} 
& U. Podemos & PSOE \\
\hline brecha laboral & 2 & 1 \\
\hline brecha salarial & 59 & 19 \\
\hline desigualdad & 21 & 24 \\
\hline discriminación & 30 & 9 \\
\hline pobreza & 16 & 1 \\
\hline precariedad & 10 & 5 \\
\hline
\end{tabular}

Una vez llevada a cabo la aproximación cuantitativa, el siguiente apartado explora en qué sentido se han empleado las voces estudiadas y qué esquemas se han activado mediante su uso.

\subsection{Aproximación cualitativa}

El estudio cuantitativo muestra que Unidos Podemos emplea con mayor frecuencia los términos analizados. Desde el punto de vista conceptual, la formación encuadra la desigualdad como un problema estructural y medular que compromete todos los órdenes de la vida. Este esquema explica el predominio de voces como patriarcado, estructural y terrorismo, menos frecuentes en el discurso socialista.

1) "Los machistas, los agresores, no son cuatro locos, las feministas decimos que son hijos sanos del patriarcado. Creo que se entiende mucho mejor si decimos que el machista es el español medio" (Rodríguez Martínez, Unidos Podemos, DS, 12/06/2018, p. 28). 
2) "Eso lo que conseguía era reproducir una estructura patriarcal que asumía roles de género según los cuales solamente las mujeres tenían que ocuparse de los cuidados" (Iglesias Turrión, Unidos Podemos, DS, 26/06/2018, p. 6).

3) “(...) significa ser conscientes de que la sociedad está cimentada en relaciones de poder, de que este problema se llama patriarcado, de que nos atraviesa al conjunto de la población, de que si tenemos que decir que nos queremos vivas es porque nuestra ciudadanía no es todavía de pleno derecho, porque nuestras vidas no están siendo libres" (Fernández Castañón, Unidos Podemos, DS, 13/09/2018, p. 27).

4) "Como decía, este es un problema estructural, es un problema que no tiene una única causa y desde luego no se soluciona solo con hacer el 'Día de...' (...)” (Fernández Castañón, Unidos Podemos, DS, 21/02/2017, p. 54).

5) "Por eso, tenemos un problema como sociedad, que se llama machismo, que es estructural, que es colectivo y hay que atajarlo con políticas públicas desde esta Cámara, que es la que legisla y la que hace posible leyes que puedan mejorar la vida de la gente. El machismo - hoy me hacían referencia a unas declaraciones que había hecho el señor Casado, también en este sentido - no tiene que ver con hombres malos; es un problema estructural en nuestro país, profundamente político y que requiere soluciones políticas" (Montero Gil, Unidos Podemos, DS, 26/02/2019, p. 18).

6) "porque entendemos que las víctimas de violencia machista han de equipararse a las víctimas de terrorismo, y no estoy diciendo que tengamos que hablar de terrorismo machista, porque esto sería perder de vista lo que es un problema estructural" (Fernández Castañón, Unidos Podemos, DS, 16/05/2017, p. 22).

7) "Si esto ocurriera con cualquier otro colectivo habrían saltado todas las alarmas, se hubieren establecido los mecanismos necesarios para frenar esta violencia y se consideraría a estas víctimas como víctimas del terrorismo. A ustedes que tanto les gusta hablar de atentados de Estado, de golpes y de víctimas del terrorismo, estas sí que son víctimas del terrorismo. Pero como son mujeres, esto no sucede" (Ramons Jordán, Unidos Podemos, DS, 23/10/2018, p. 24).

La voz cuidados, también característica de su discurso y menos empleada por el PSOE, se importa del campo teórico de la filosofía. Con ella se defiende que las mujeres construyen los razonamientos morales a partir de lo relacional, es decir, desde el vínculo con los demás (Gilligan, 1982). Esta asimetría está en la base de la división sexual del trabajo. La voz, cada vez más presente en el discurso político, se utiliza en referencia a las tareas del ámbito doméstico (cuidado de niños, ancianos, personas dependientes, etc.), que realizan mayoritariamente mujeres. Participa de un marco que busca concienciar sobre el trabajo doméstico no remunerado.

8) "sabemos que en los países en los que esto se ha puesto en marcha, países de referencia muchas veces en esta sala como Suecia o Islandia, se demuestra que los padres toman esos permisos y la sociedad allí avanza en cómo entiende los cuidados y en cómo entiende la igualdad" (Fernández Castañón, Unidos Podemos, DS, 18/10/2016, p. 44).

9) “Tenemos claro que los cuidados tienen que estar repartidos, y decimos palabras como conciliación y decimos palabras como corresponsabilidad" (Fernández Castañón, Unidos Podemos, DS, 27/06/2017, p. 55). 
10) "Por eso, cuando hablamos de repartir los cuidados no solamente hablamos de una cuestión moral o ideológica; es una cuestión también económica, también política, de vértebra del sistema político, económico y social español, de convivencia" (Montero Gil, Unidos Podemos, DS, 20/02/2018, p. 7).

En el plano discursivo, ambas formaciones utilizan el denominado lenguaje inclusivo, aunque es una estrategia más habitual en el discurso del partido morado. En los debates parlamentarios sobre igualdad los diputados desdoblan los sustantivos con el objetivo de visibilizar a la ciudadanía femenina. El intento de eliminar supuestos sesgos androcéntricos en el lenguaje es común a las formaciones de izquierda ${ }^{6}$. La Real Academia Española (RAE) defiende, no obstante, una posición tradicional al respecto y censura el uso de este lenguaje. Solo considera justificado el desdoblamiento en los contextos en los que puede haber ambigüedad (NGLE, 2009, § 2.2.), como en No tiene hermanos ni hermanas, pero rechaza que el empleo del masculino genérico lleve aparejada una discriminación lingüística. En el lenguaje político, se suelen desdoblar los sustantivos que se refieren a personas como estrategia comunicativa para demostrar un compromiso igualitario. A continuación, pueden verse algunos ejemplos:

11) "que voten hoy pensando en sus hijos o hijas, incluso ustedes han sido alguna vez hijo o hija” (Fernández Castañón, Unidos Podemos, DS, 18/10/2016, p. 45).

12) "que muchas y muchos en algún momento hemos tenido una conversación entre amigas o amigos o quizá con la familia y nos puede haber salido algún argumento 'cuñado' en el que se diga que esto de la brecha salarial no debe ser verdad" (Fernández Castañón, Unidos Podemos, DS, 21/02/2017, p. 53).

13) "cultura eminentemente adultocéntrica que sigue sin considerar a los niños y las niñas como sujetos, como ciudadanos y ciudadanas de pleno derecho" (Belarra Urteaga, Unidos Podemos, DS, 16/05/2017, p. 23).

14) "No todos los cuidadores o cuidadoras o los que se dedican a la crianza son padres o madres" (Guinart Moreno, PSOE, DS, 27/06/2017, p. 58).

15) "[...] se fraguó vinculando a todos $\mathbf{y}$ a todas en un compromiso no solo contra la violencia de género sino también contra el sexismo que la alimenta" (Álvarez Álvarez, PSOE, DS, 15/11/2016, p. 34).

Un caso más problemático es el del pronombre nosotros. La RAE explica en su informe sobre lenguaje inclusivo (2020) que el desdoblamiento de los pronombres personales entraña algunas dificultades. La sustitución de nosotros por nosotros y nosotras, cada vez más frecuente en el discurso político, solo es equivalente en uno de los doce supuestos (RAE, 2020, § 8.3.4.3). Desde el punto de vista deíctico, carece de sentido: si nosotros se refiere al masculino y nosotras al femenino, un orador varón debería decir "nosotros y vosotras", y viceversa una oradora. Si se entienden, en cambio, como pronombres genéricos, entonces no tiene sentido desdoblarlos. Quienes emplean la construcción desdoblada o bien pasan por

\footnotetext{
${ }^{6}$ Todavía hoy resuena la expresión "miembros y miembras", empleada en 2008 por Bibiana Aído (ministra de Igualdad del gobierno socialista hasta 2010); y más aún, por estar más cercana en el tiempo, la de Irene Montero, que incorporó en un discurso de 2018 el término portavozas.
} 
alto esta reflexión o bien lo hacen estratégicamente con el fin de demostrar su compromiso político.

Como se apuntó en la aproximación cuantitativa, nosotras es una de las voces que caracterizan el discurso de la formación morada. Se utiliza en ocasiones como femenino genérico, como muestran los dos últimos ejemplos:

16) "Por supuesto nosotros y nosotras vamos a apostar, como siempre, por una atención psicológica de calidad" (Belarra Urteaga, DS, 16/05/2017, p. 23).

17) "Por eso empiezo a pensar que este concepto griego de la parresía que tendría que estar impregnando la Cámara del Congreso no sirve de nada, porque no nos escuchamos ni siquiera cuando se dirigen a nosotros y a nosotras" (Fernández Castañón, DS, 09/05/2018, p. 48).

18) “¿Dónde está el «si nos tocan a una nos tocan a todas»? ¿Me puede decir dónde está? Porque de eso es de lo que va el feminismo. Nosotras defendemos un feminismo en el que quepamos todas, y todas somos todas" (Fernández Castañón, DS, 19/02/2019, p. 39).

19) "No hay mejor forma para nosotras de terminar esta legislatura que presentando esta PL para garantizar las libertades sexuales de todas las mujeres" (Montero Gil, DS, 26/02/2019, p. 17).

Por último, los términos violencias, miedo y violación destacan en el discurso del partido morado. Se utilizan para vincular el debate sobre igualdad de género con un campo semántico que remite al sufrimiento y a la inseguridad que viven las mujeres. Unidos Podemos crea así un marco que incide en las muertes, los asesinatos, las violaciones, las agresiones, el miedo y las violencias. Esta última voz, en plural, es empleada casi de manera exclusiva por la formación para hacer alusión a todas las formas de discriminación sexista.

20) "Se acabó el tiempo de la muerte, el tiempo de los asesinatos; queremos un tiempo nuevo y feminista en el que no tengamos mujeres con miedo" (Díaz Pérez, DS, 12/06/2018, p. 23).

21) "políticas feministas para que vivamos sin miedo, para que vivamos de pleno derecho todas las mujeres, todas, las migrantes, las racializadas, las mujeres lesbianas, las mujeres trans, las precarias, las que tienen diversidad funcional, todas las mujeres de este país" (Fernández Castañón, DS, 09/05/2018, p. 44).

22) "Cuando a las mujeres las están violando, agrediendo, discriminando y matando, lo que hace la buena intención es chirriarnos.” (Fernández Castañón, DS, 09/05/2018, p. 43).

23) "El machismo no es un accidente, no es una lacra, no es algo inevitable; el machismo, precisamente, es lo que está asesinando, violando, agrediendo y discriminando a las mujeres en este país. No se trata de sesgos porque esto es una cuestión medular, que está en la médula de nuestra sociedad" (Fernández Castañón, DS, 28/09/2017, p. $31)$.

24) "Estamos hablando de muchas más violencias que aquellas que se ejercen en el ámbito de la pareja o de la expareja. Por tanto, llamémosla violencia machista, y no porque sea un capricho nuestro, sino porque es importante que nombremos aquellas cosas que queremos combatir, porque las realidades que no son nombradas no existen" (Rodríguez Martínez, DS, 15/11/2016, p. 38). 
25) "Este es el último Pleno de la legislatura y esta ley para garantizar las libertades sexuales y combatir las violencias sexuales hacia las mujeres es la última proposición de ley que mi grupo parlamentario va a presentar"; "Nosotras queremos erradicar las violencias sexuales, queremos erradicar las violencias machistas en todos los ámbitos de la vida e independientemente de la relación de la víctima con el agresor" (Montero Gil, DS, 26/02/2019, p. 18).

En lo que se refiere a los marcos difundidos por el PSOE, son similares a los de Unidos Podemos. Se subraya la lacra que supone el machismo para la sociedad, aunque no aparece conceptualizado generalmente como un problema estructural. La construcción brecha salarial, menos frecuente que en el corpus morado, se emplea con un sentido similar. En cuanto a los términos relacionados con la conciliación, el PSOE utiliza el mismo esquema mental para hablar de la corresponsabilidad y los cuidados que U. Podemos, si bien emplea este término, como se vio en la parte cuantitativa, solo en siete ocasiones, frente a las cuarenta y nueve del partido morado.

26) "La cuestión es que con cuarenta y cuatro asesinatos de mujeres, de ocho niños y niñas y con veintidós menores huérfanos por culpa del machismo criminal esperamos que nadie dude que esos 200 millones deben estar en ese catálogo [...]" (Álvarez Álvarez, PSOE, DS, 21/11/2017, p. 32).

27) "La brecha salarial es la manifestación más clara de la desigualdad." (Rodríguez García, PSOE, DS, 15/02/2017, p. 28).

28) "[...] darnos cuenta de que no estamos ante una brecha sino ante un abismo que necesita de urgente acción política" (Rodríguez García, PSOE, DS, 15/02/2017, p. $32)$.

29) "El Estado debe asumir su corresponsabilidad en los cuidados, pero también en el fomento de la corresponsabilidad en el ámbito privado y empresarial, y debe hacerlo porque efectivamente, señorías, es viable, es beneficioso socialmente y es un elemento clave para la igualdad" (Álvarez Álvarez, PSOE, DS, 18/10/2016, p. 48).

El análisis pone de manifiesto que las diferencias entre las dos formaciones son, por lo general, cuantitativas. U. Podemos emplea de forma más habitual la mayor parte de los términos analizados. Desde el punto de vista cualitativo, ambos partidos utilizan, sin embargo, marcos similares, si bien la formación morada insiste más en el carácter estructural de la desigualdad y emplea de manera exclusiva palabras como patriarcado, violencias o terrorismo.

\section{Conclusiones}

Este estudio da cuenta de la selección léxica empleada por los partidos progresistas de ámbito nacional en los debates parlamentarios sobre igualdad de género durante la XII legislatura (2016-2019). La aproximación cuantitativa pone de manifiesto que existen diferencias en el uso de los términos. Unidos Podemos emplea con mayor frecuencia que el PSOE las voces analizadas; destaca en el uso de palabras como feminismo, patriarcado, cuidados o violencias. Las utiliza para denunciar que el machismo sea un problema sistémico que compromete los derechos de las mujeres en todos los órdenes.

Mientras el partido morado emplea de manera similar las voces feminismo e igualdad, en el discurso de los socialistas se atestigua una preferencia clara por la 
segunda en detrimento de la primera, lo que indica que el sustantivo feminismo continúa estando más marcado. Los términos patriarcado, violencias, terrorismo y cuidados también corresponden a Unidos Podemos; no aparecen en ninguna de las intervenciones parlamentarias del PSOE. En cuanto a voces como machismo, estructural o revolución, están presentes en ambos corpus con un sentido similar, aunque predominan en el del partido situado más a la izquierda.

El campo semántico relativo a la inseguridad que sufren las mujeres también es más explotado por la formación morada, que emplea con mayor frecuencia voces como acoso, agresión, asesinato, miedo, violación o violencia. En el léxico relacionado con las condiciones laborales hay menos disparidad (por ejemplo, en el empleo de términos como conciliación o corresponsabilidad apenas hay diferencia), aunque Unidos Podemos sobresale en el uso de construcciones como brecha salarial, discriminación o precariedad. También utiliza de manera más habitual los sustantivos mujeres y nosotras. En cuanto al análisis cualitativo, destaca Unidos Podemos por el uso más frecuente del lenguaje inclusivo y por una conceptualización que entiende la desigualdad como un problema estructural que afecta a todas las esferas (económica, política, doméstica, sexual, etc.).

Será labor de trabajos posteriores determinar qué uso hacen de las voces implicadas las fuerzas conservadoras, así como ampliar la nómina de términos y ver su distribución en el tiempo, a fin de tener una comprensión más global sobre el discurso político que se elabora en torno a la igualdad de género. Esperamos que este estudio preliminar pueda ser tomado como punto de partida para acometer las futuras investigaciones.

\section{Referencias}

1. Actas del Congreso de los Diputados de la XII legislatura (2016-2019). Disponibles en la Página Web del Congreso de los Diputados [última consulta: 15-12-2020].

2. Ardèvol-Abreu, A. (2015): "Framing o teoría del encuadre en comunicación. Orígenes, desarrollo y panorama actual en España". Revista Latina de Comunicación Social, 70, pp. 423 a 450. http://www.revistalatinacs.org/070/paper/1053/23es.html https://doi.org/10.4185/RLCS-2015-1053

3. Arrieta Castillo, Carolina. (2017). Los discursos persuasivos de progresistas y conservadores en el debate sobre el acceso de los homosexuales al matrimonio. Tesis doctoral. Salamanca: Universidad de Salamanca.

4. Arrieta Castillo, C. (2021). La semántica cognitiva y las representaciones LGTBI+. Viejos discursos desde nuevas perspectivas. En Puig Guisado, J., Torres Fernández, C. e Iglesias Redondo, M. R. (Eds). Análisis y propuestas educativas sobre género y diversidad sexual: Sociedades y escrituras en continuas transformaciones. (pp. 115130). Madrid: Dykinson. https://doi.org/10.2307/j.ctv1s7cjkn.9

5. De Santiago Guervós, J. (1992). El léxico político de la transición española. Salamanca: Ediciones Universidad de Salamanca.

6. De Santiago Guervós, J. (2005). Principios de comunicación persuasiva. Madrid: Arco Libros.

7. Del Rey Morató, J. (2011). La comunicación política en la sociedad del marketing y de internet. Encuadres, relatos y juegos de lenguaje, Revista de Comunicación, 10, pp. 102-128.

8. Fernández Lagunilla, M. (1999a). La lengua en la comunicación política I: el discurso del poder. Madrid: Arco Libros.

9. Fernández Lagunilla, M.. (1999b). La lengua en la comunicación politica II: el discurso del poder. Madrid: Arco Libros. 
10. Francesconi, A. (2012). El sexismo en el lenguaje político español e italiano. Nómadas: Critical Journal of Social and Juridical Sciences, 34, pp. 139-147. https://doi.org/10.5209/rev_NOMA.2012.v34.n2.40736

11. Fuentes, C. (2015). Seminario Discurso y sociedad: la representación del género en el discurso. Discurso y Sociedad, 9 (1/2), pp. 100-105.

12. Gallardo Paúls, B. (2014). Usos políticos del lenguaje. Un discurso paradójico. Barcelona: Anthropos Editorial.

13. García Santos, J. F. (1980). Léxico y política de la segunda república española. Salamanca: Ediciones Universidad de Salamanca.

14. García Santos, J. F. (1982). El léxico de la Segunda República. Boletín Informativo Fundación Juan March, 111, pp. 41-42.

15. Lakoff, G. y Johnson, M. (1987). The Metaphorical Logic of Rape. Metaphor \& Symbolic Activity, 2 (1), pp. 73-79. https://doi.org/10.1207/s15327868ms0201_5

16. Lakoff, G. (2004). Don't think of an elephant! Know your values and frame the debate: the essential guide for progressives. Vermont: Chelsea Green Publishing.

17. Litosseliti, L. y Sunderland, J. (Eds.) (2002). Gender Identity and Discourse Analysis. Londres: John Benjamins Publishing. https://doi.org/10.1075/dapsac.2

18. López Eire, A. y De Santiago Guervós, J. (2000). Retórica y comunicación política. Madrid: Cátedra.

19. Martín Rojo, L. y Van Dijk, T. A. (1997). 'Había un problema y se ha solucionado'. La legitimación de la expulsión de inmigrantes 'ilegales' en el discurso parlamentario español. Discourse and Society, 8 (4), pp. 523-566.

20. Núñez Cabezas, E. A. (2000). Aproximación al léxico del lenguaje político español. Tesis doctoral. Málaga: Universidad de Málaga.

21. Núñez Cabezas, E. A. y Guerrero Salazar, S. (2002). El lenguaje politico español. Madrid: Cátedra.

22. Olmos, A. (2012). Discurso político e inmigración: análisis crítico de discurso a propósito de la reforma gubernamental realizada al sistema de salud pública en España. Discurso y Sociedad, 6 (4), pp. 739-758.

23. Panncake, A. S. (1993). Taken by Storm: The Exploitation of Metaphor in Persian Gulf War. Metaphor \& Symbolic Activity, 8 (4), pp. 281-295. https://doi.org/10.1207/s15327868ms0804_2

24. Real Academia Española (2009). Nueva gramática de la lengua española. Madrid: Espasa.

25. Real Academia Española. (2020). Informe de la Real Academia Española sobre el uso del lenguaje inclusivo en la Constitución Española, elaborado a petición de la Vicepresidenta del Gobierno. Boletín de Información Lingüística de la Real Academia Española, 14.

26. Tannen, D. (1994). Gender and discourse. Nueva York: Oxford University Press.

27. Van Dijk, T. A. (2001). Critical discourse analysis. En Schiffrin, D., Tannen D. y Hamilton, H. (Eds.). The Handbook of Discourse Analysis, pp. 352-371. Oxford: Blackwell.

28. Wodak, R. (2003 [2001]). De qué trata el análisis crítico del discurso (ACD). Resumen de su historia, sus conceptos fundamentales y sus desarrollos. En Wodak, R. y Meyer, M. (Eds.). Métodos de análisis crítico del discurso, pp. 17-34. Barcelona: Editorial Gedisa.

29. Zapata-Barrero, R. (2007). Política del discurso sobre inmigración en España: discurso reactivo y discurso pro-activo en los debates parlamentarios. Discurso y Sociedad, 2 (1), pp. 317-369. 